\title{
Endoscopia en pancreatitis crónica
}

\author{
Jorge A. López-Cossío, Everardo Muñoz-Anaya y Félix I. Téllez-Avila* \\ Departamento de Endoscopia, Instituto Nacional de Ciencias Médicas y Nutrición Salvador Zubirán, Ciudad de México, México
}

\section{Resumen}

Hasta hace poco tiempo el papel de la endoscopia en pacientes con pancreatitis crónica estaba limitado a un papel en el diagnóstico y generalmente solo se lograba en estadios avanzados de la enfermedad cuando ya existían cambios evidentes por otros métodos de estudio no invasivos. Por otro lado, el papel en el tratamiento también era limitado y con resultădos distantes de ser óptimos, principalmente esto debido a la elección inadecuada de los pacientes.

Palabras clave: Endoscopia. Pancreatitis crónica. CPRE. Ultrasonido endoscópico.

\section{Endoscopy in chronic pancreatitis}

\begin{abstract} patients.

Key words: Endoscopy. ERCP. Endoscopic ultrasound. Chronic pancreatitis.
\end{abstract}

Until recently, the role of endoscopy in patients with chronic pancreatitis was limited only for diagnosis and generally, itwas reach in advanced stages of the disease when the changes were evident in other non-invasive methods. Regarding treatment, the role of endoscopy was very limited too with questionable results, it was related mainly to inadequate selection of the

\section{Introducción}

La pancreatitis crónica es una patología compleja observada con frecuencia en gastroenterología, su incidencia anual es de 4 casos por cada 100,000 personas-año y se caracteriza por inflamación crónica y fibrosis del páncreas. Las complicaciones tardías relacionadas con esta entidad incluyen estenosis del conducto pancreático, litiasis pancreática, pseudoquiste pancreático, fístulas y dolor abdominal crónico, en ocasiones es necesario realizar un manejo multidisciplinario y multimodal para el alivio de los síntomas incluyendo endoscopia, radiología intervencionista y cirugía pancreática ${ }^{1}$.

La terapia endoscópica en pancreatitis crónică va dirigida principalmente al alivio del dolor abdominâal y el manejo de complicaciones. El dolor abdominal àsociado a pancreatitis crónica puede abarcar dessde molestias inespecíficas posprandiales hasta un dolor incapacitante. Se han propuesto diversos mecanismos para explicar el dolor abdominal en pancreatitis crónica, por vía de estímulos nociceptivos de origen višceral, anormalidades estructurales provocadas poof la
Correspondencia:

*Félix I. Téllez-Ávila

E-mail: felixtelleza@gmail.com
Fecha de recepción: 01-06-2019

Fecha de aceptación: 07-08-2019

DOI: 10.24875/END.M19000172

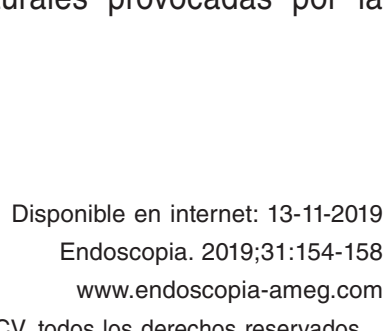


obstrucción del conducto pancreático y el daño neurológico secundario a inflamación y fibrosis, por lo tanto el manejo endoscópico puede dirigirse a diferentes blancos terapéuticos. Los abordajes de tratamiento endoscópico para el dolor de origen pancreático incluyen el bloqueo del plexo celiaco, drenaje endoscópico de pseudoquiste pancreático, descompresión del conducto pancreático y tratamiento de estenosis biliares benignas secundarias a pancreatitis crónica, las cuales se revisarán a continuación².

\section{Manejo del dolor}

\section{Bloqueo del plexo celiaco guiado por ultrasonido endoscópico}

El plexo celiaco se localiza anterolateral a la aorta a nivel de L1, se encuentra conformado por diferentes ganglios incluyendo ganglios aórticos, renales y mesentéricos; en él se combinan fibras nerviosas del sistema simpático y parasimpático. Diversos órganos abdominales reciben inervación por vía del plexo celiaco incluyendo el hígado, páncreas, vesícula biliar, estómago, bazo, riñones, intestino delgado y $2 / 3$ del colon proximal. El dolor en pancreatitis crónica puede ser visceral (en el $22 \%$ ) y no visceral (en el $78 \%$ ), lo cual explica la diversidad en la respuesta al tratamiento ${ }^{3}$. El bloqueo del plexo celiaco es un procedimiento de segunda línea para el manejo del dolor en pancreatitis crónica, se realiza guiado por ultrasonido endoscópico (USE), este método ha demostrado ser superior al procedimiento guiado por tomografía computarizada o fluoroscopia ${ }^{4}$. Se han evaluado estudios acerca de la eficacia del bloqueo del plexo celiaco, los estudios actuales presentan variabilidad en la forma de evaluar la respuesta al tratamiento, en promedio se espera una respuesta del $60-70 \%$ con mejoría de los síntomas además de reducir la dependencia al uso de narcóticos, con duración de la respuesta desde 1 semana a 1 mes y pérdida posterior del efecto analgésico; se ha observado una mejor respuesta en pacientes de edad avanzada y aquellos pacientes que responden en la primera sesión de bloqueo del plexo. Mediante USE se localiza la emergencia del tronco celiaco, posteriormente se inyectan anestésicos locales y esteroides que inhiben temporalmente el plexo, reducen la inflamación y mejoran la calidad de vida ${ }^{5}$. Existen dos técnicas para la administración de los fármacos, la inyección bilateral o unilateral y la inyección directa al ganglio celiaco, sin embargo no se ha encontrado una diferencia significativa entre ellas. Los fármacos utilizados son bupivacaína
$20 \mathrm{ml}$ al $25 \%$ con $80 \mathrm{mg} \mathrm{(4} \mathrm{ml)} \mathrm{de} \mathrm{triamcinolona}{ }^{6}$. El procedimiento se encuentra contraindicado en pacjentes con inmunocompromiso por el riesgo de infección y en coagulopatía o trombocitopenia, que incrementan el riesgo de hemorragia. Dentro de los eventos adversos se incluye diarrea (1-20\%), hipotensión ortostâtica (1-20\%), exacerbación del dolor (en el 9\%) a 48 horas, infección y hemorragia; una complicación infrecuente es la lesión a la arteria de Adamkiewicicz, que irriga el segmento medular anterior y puede lèvar al síndrome de la arteria espinal anterior?.

\section{Descompresión del conducto pancreático}

La obstrucción del conducto pancreático puede presentarse secundario a litiasis pancreática, estenôsis del conducto pancreático y/o compresión del condūcto pancreático por un pseudotumor o pseudoquiste. pancreático. La descompresión del conducto pancreático se lleva a cabo mediante la realización de los sigưientes procedimientos: 1) esfinterotomía pancreática, 2) colocación de prótesis pancreática, y 3) extracceión de litos en el conducto pancreático.

\section{Esfinterotomía pancreática}

Mediante la esfinterotomía pancreática se descomäprime el conducto pancreático. Este procedimiento permite el acceso al conducto pancreático para realizar futuros procedimientos terapéuticos, en caso de obstrucción ampular puede considerarse la esfinterotomía pancreática como monoterapia. El procedimiento se puede realizar de dos maneras, canulando la papila mayormediante el uso de un esfinterotomo, dirigiendo el accesorio y realizando el corte hacia la posición 1 de las manecillas del reloj; la segunda técnica se realiza con aguja cortante, ya sea colocando o no una prótesis plástica panç̄eática previo al corte ${ }^{8}$. Un estudio aleatorizado demostró resultados a favor de realizar la esfinterotomía con ağuja cortante sobre una prótesis plástica, ya que este método se asoció a una menor tasa de complicaciones, en particular pancreatitis post-Colangiopancreatografía rêtrógrada endoscópica (CPRE) comparado con la técnica estándar 0 y $29 \%$, sin embargo esta técnica se ha ă̌sociado a reestenosis de la esfinterotomía en el 15-30\% en algunas series de $\operatorname{casos}^{9}$.

\section{Endoprótesis pancreática}

Las prótesis pancreáticas se utilizan para el tratamiento de las estenosis del conducto pancreático, 


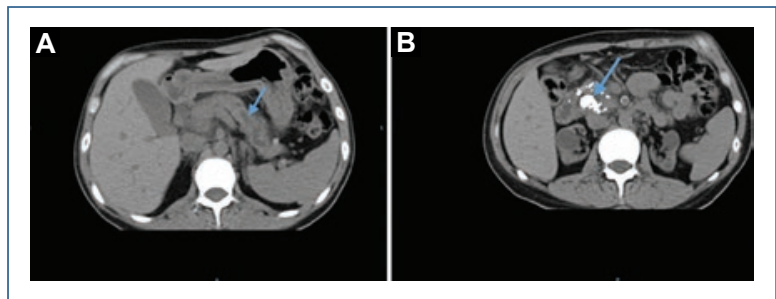

Figura 1. A: tomografía simple de abdomen donde se observa el conducto pancreático dilatado a nivel de cuerpo y cola de páncreas (flecha). B: tomografía computarizada simple de abdomen donde se observan calificaciones a nivel de la cabeza del páncreas (flecha).

fugas o en caso de litiasis pancreática no resuelta. La respuesta al tratamiento suele ser favorable, sin embargo la respuesta a largo plazo es subóptima. Se utilizan prótesis de 3-5 Fr y en casos en los cuales el conducto pancreático se encuentra dilatado se utilizan prótesis de 7-10 $\mathrm{Fr}^{10}$. En pacientes con estenosis recurrentes se utilizan múltiples prótesis plásticas para dilatar la estenosis. Actualmente existe poca información acerca de cuánto tiempo debe permanecer colocada la prótesis pancreática. Existen diferentes tipos de prótesis plásticas, con variaciones en el calibre y el material. Las prótesis metálicas totalmente cubiertas se han evaluado en el tratamiento de estenosis del conducto pancreático. Una preocupación con este tipo de prótesis es la obstrucción de conductos secundarios debido a la cobertura de la prótesis, la respuesta se ha reportado de hasta el $80-100 \%$ con este tipo de prótesis, sin embargo el seguimiento ha sido a corto plazo (5 meses) ${ }^{11,12}$. En la actualidad la evidencia es a favor de utilizar prótesis plásticas de mayor calibre, basado en un estudio que evaluó la colocación de múltiples prótesis plásticas en el conducto pancreático con un seguimiento de 38 meses, con un $84 \%$ de los pacientes encontrándose asintomáticos ${ }^{13}$.

\section{Extracción de lito y litotricia endoscópica}

Los litos en el conducto pancreático pueden provocar obstrucción y aumento de la presión intraductal activando la cascada inflamatoria, lo cual lleva a mayor fibrosis (Ver Figura 1). Existen diversos métodos para la extracción del lito, se puede realizar por vía retrógrada utilizando canastillas de Dormia, balones extractores o fórceps, los factores que se asocian a mayor éxito son litos pequeños, móviles, sin estenosis del conducto pancreático. La extracción de litos $>5 \mathrm{~mm}$ puede no ser posible con técnicas estándar, y en casos donde existe un lito impactado, se utilizan la litotricia extracorpórea (LTEC) y la litotricia intraductal.

La LTEC es otro método útil en litos $>5 \mathrm{~mm}$, litos impactados o aquellos localizados detrás de una zona de estenosis, destruye los litos pancreáticos en el $\overline{9} 0 \%$ de los casos y se asocia con aclaramiento espontâneo en el $80 \%$ sin necesidad de endoscopia, siendo elobjetivo una fragmentación $<3 \mathrm{~mm}^{14}$.

La litotricia a través del endoscopio consiste en destruir los litos con catéteres especiales mediannte pancreatoscopia. Existen dos tipos de litotricia endoscópica, la electrohidráulica y la litotricia láser, cợ la posibilidad de mantener visión directa en el condưcto pancreático reduciendo complicaciones como hemorragia y perforación. Un estudio reciente evaluQ̊ la seguridad y eficacia de la pancreatografía retrógráda endoscópica (PRE) y la pancreatoscopia comparado con PRE, encontrando mayor éxito técnico (98.9uvs. $87.6 \%$ ) en aquellos pacientes en los que realizó pancreatoscopia permitiendo la extracción efectiva dêlos litos cuando la técnica estándar no fue posible ${ }^{15}$.

\section{Drenaje de pseudoquiste pancreático}

El pseudoquiste pancreático es una complicación que se desarrolla en el $20-40 \%$ de los pacientes pancreatitis crónica, a diferencia de los pacientes con pancreatitis aguda solo una minoría de estos pseưdoquistes resuelven de manera espontánea ${ }^{16}$.

La terapia endoscópica es el procedimiento de elección para el tratamiento de los pseudoquistes, con resultados comparables con la cirugía, asociados a menor costo, menor estancia hospitalaria y mejoria en la calidad de vida. Las indicaciones de tratamiento incluyen dolor abdominal, saciedad temprana, pérđida de peso, náuseas y vómitos asociados a obstrucción el tracto de salida gástrico, ictericia e infección; también se pueden considerar en pacientes asintomáticos con un pseudoquiste $>5 \mathrm{~cm}$ que no resuelve de $\mathrm{ma}$ nera espontánea tras 3-6 meses de seguimiento. Existen dos métodos para realizar el drenaje del pšeudoquiste: el drenaje transpapilar y el drenaje transmural, este último idealmente se realiza guiado por USE ${ }^{17}$. La European Society of Gastrointestinal Endoscopy (ESGE) recomienda el uso de múltiples prótesis ptásticas doble cola de cochino para realizar el drenaje con un mínimo de tiempo de 2 meses hasta la resolueieión del quiste, ya que el retiro prematuro se asocia a recurrencia; el éxito clínico se define como una reducoción del $30-50 \%$ en el diámetro de la colección en 1 mes, 
logrando un éxito técnico más del $90 \%$, las complicaciones ocurren en el $13 \%$ con mortalidad del $0.3 \%$.

\section{Drenaje transpapilar}

Se recomienda cuando la colección se encuentre comunicada con el conducto pancreático principal y el quiste mida $<6 \mathrm{~cm}$ de diámetro, manteniendo un drenaje por un periodo de 4-6 semanas para pseudoquistes pequeños localizados en cabeza o cuerpo de páncreas con un éxito del $80 \%$. Se prefiere el uso de prótesis plásticas doble cola de cochino $10 \mathrm{Fr}$ para prevenir la migración de la prótesis ${ }^{18}$.

\section{Drenaje transmural}

En casos donde el pseudoquiste no comunica con el conducto pancreático se puede realizar por endoscopia una cistogastrostomía o cistoduodenostomía. Si se visualiza compresión del pseudoquiste se puede realizar por vía endoscópica, la localización del pseudoquiste debe $\mathrm{ser}<1-1.5 \mathrm{~cm}$ de distancia de la pared gástrica. EI USE se encuentra indicado en casos de hipertensión portal o ausencia de compresión visible por vía endoscópica para evitar complicaciones, el éxito del USE ha sido reportado en el 88-95\%. Durante el procedimiento se localiza el sitio de punción y posteriormente se utiliza coagulación y un balón dilatador para crear el trayecto y colocar una prótesis plástica o metálica con un éxito del $95 \%$ a los 3 meses $^{17,18}$. El uso de prótesis metálicas autoexpandibles o prótesis de aposición luminal no ha demostrado superioridad en el drenaje del pseudoquiste, la ventaja que demuestran sobre las prótesis plásticas es que se puede crear un diámetro de drenaje mayor reduciendo el tiempo que lleva para la resolución del pseudoquiste y menor oclusión de la prótesis, las desventajas incluyen la hemorragia y migración de la prótesis ${ }^{19}$. El uso de prótesis metálicas con sistema antimigración podría evitar esta última complicación ${ }^{20}$.

\section{Estenosis biliares asociadas a pancreatitis crónica}

Las estenosis biliares intrapancreáticas se encuentran en el $3-46 \%$ de los pacientes con pancreatitis crónica, esta complicación ocurre secundaria a fibrosis 0 compresión extrínseca por colecciones líquidas peripancreáticas, y puede llevar a colangitis, litiasis biliar y cirrosis biliar secundaria en el $10 \%$ de los casos. Cuando son diagnosticadas es importante descartar la presencia de un proceso neoplásico ${ }^{21}$. El tratamiento consiste en colocar una endoprótesis biliar plástica o metálica para mantener el drenaje biliar. Se ha demostrado en estudios que este tipo de estenosis pueden ser refractarias al tratamiento, la colocación de ựna endoprótesis biliar plástica se asocia a altas tasaș de falla terapéutica, un estudio no aleatorizado demostró éxito clínico del 24 vs. 92\% colocando una prótesis plástica vs. múltiples prótesis plásticas. Por este mòtivo la recomendación actual es utilizar múltiples prótessis plásticas con recambios cada 3 meses y duracióñolel tratamiento mínimo de 1 año ${ }^{22}$. En cuanto a las prótesis metálicas un estudio comparó estas prótesis con elưuso de prótesis plásticas demostrando no inferioridad La ESGE recomienda la colocación de una endoprótesis biliar en estenosis sintomáticas, cirrosis biliar secuñdaria o elevación asintomática de la fosfatasa alcaliñ > 2-3 veces límite superior normal o elevación de bilitrubina total > 1 mes. Continúa en estudio cuál es la prótesis ideal para el tratamiento de estenosis biliares, la duración y los recambios de prótesis necesarios ${ }^{23}$ ?

\section{Conclusiones}

En conclusión, los avances tecnológicos y la mejoría en las técnicas endoscópicas han permitido expandir la capacidad de tratamiento en pancreatitis crónica sin embargo en la actualidad continúa siendo un reto para el gastroenterólogo. El manejo endoscópico permanece como el tratamiento de primera línea en las diversas complicaciones asociadas a la enfermedad, sin embargo es importante recordar que se requiere de un equipo multidisciplinario para el adecuado manejo de éste grupo de pacientes.

\section{Bibliografía}

1. Majumder S, Chari ST. Chronic pancreatitis. Lancet. 2016;387:1957-66.

2. Pasricha PJ. Unraveling the mystery of pain in chronic pancreatitis. Nat Rev Gastroenterol Hepatol. 2012;9:140-51.

3. Conwell DL, Vargo JJ, Zuccaro G, et al. Role of differential neuroaxial blockade in the evaluation and management of pain in chronic pancreatitis. Am J Gastroenterol. 2001;96(2):431-6.

4. Gress $F$, Schmitt $C$, Sherman S, et al. A prospective randomized comparison of endoscopic ultrasound and computed tomography-guided celiac plexus block for managing chronic pancreatitis pain. Am J Gastroenterol. 1999;94:900-5.

5. Levy MJ, Topazian MD, Wiersema MJ, et al. Initial evaluation of the efficacy and safety of endoscopic ultrasound-guided direct ganglianeurolysis and block. Am J Gastroenterol. 2008;103:98-103.

6. LeBlan JK, DeWitt J, Johnson C, et al. A prospective randomized trial of 1 versus 2 injections during EUS-guided celiac plexus block for chronic pancreatitis pain. Gastrointest Endosc. 2009;69:835-42.

7. Sey M, Schmaltz SL, Al-Haddad L, et al. Effectiveness and safety of serial endoscopic ultrasound-guided celiac plexus block for chronic-pancreatitis. Endosc Int Open. 2015;3(1):E56-9.

8. Attwell A, Borak G, Hawes $R$, et al. Endoscopic pancreatic sphincterotomy for pancreas divisum by using a needle-knife or standard püf-type technique: safety and reintervention rates. Gastrointest Endosc. 2006;64(5):705-11. 
9. Varadarajulu S, Wilcox C. Randomized trial comparing needle-knife and pull-sphincterotome techniques for pancreatic sphincterotomy in high-risk patients. Gastrointest Endosc. 2006;64(5):716-22.

10. Moon SH, Kim MH, Park DH, et al. Modified fully covered self-expandable metal stents with antimigration features for benign pancreatic-duct strictures in advanced chronic pancreatitis, with a focus on the safety profile and reducing migration. Gastrointest Endosc. 2010;72(1):86-91.

11. Ogura T, Onda S, Takagi $W$, et al. Placement of a $6 \mathrm{~mm}$, fully covered metal stent for main pancreatic head duct structure due to chronic pancreatitis: a pilot study. Therap Adv Gastroenterol. 2016;9(5):722-8.

12. Park D, Kim M, Moon S, et al. Feasibility and safety of placement of a newly designed, fully covered self-expandable metal stent for refractory benign pancreatic ductal strictures: A pilot study. Gastrointest Endosc 2008;68:1182-9.

13. Costamagna G, Bulajic M, Tringali A, et al. Múltiple stenting of refractory pancreatic duct strictures in severe chronic pancreatitis: long-term results. Endoscopy. 2006;38(3):254-9.

14. Moole H, Jaeger A, Bechtold ML, et al. Success of extracorporeal shock wave lithotripsy in chronic calcific pancreatitis management: A meta-analysis and systematic review. Pancreas. 2016;45(5):651-8.

15. Han S, Shah RJ, Brauer BC, et al. A comparison of endoscopic retrograde pancreatography with or without pancreatoscopy for removal of pancreatic duct stones. Pancreas. 2019;48(5):690-7.
16. Andren-Sandberg A, Dervenis C. Pancreatic pseudocysts in the $21^{\text {st }}$ century. Part I: Classification, pathophysiology, anatomic considerations and treatment. JOP. 2004;5(1):8-24.

17. Krüger M, Schneider AS, Manns MP, et al. Endoscopic management of pancreatic pseudocysts or abcesses after an EUS guided 1-step procedure for initial access. Gastrointest Endosc. 2006;63(3):409-16.

18. Kahaleh M, Shami VM, Conaway MR, et al. Endoscopic ultrasound drainage of pancreatic pseudocyst: a prospective comparison withconventional endoscopic drainage. Endoscopy. 2006;38(4):355-9.

19. Itoi T, Binmoeller KF, Shah J, et al. Clinical evaluation of a novel lumen-apposing metal stent for endosonography-guided pancreatic pseudocyst and gallbladder drainage. Gastrointest Endosc. 2012;75(4):870-6.

20. Téllez-Ávila FI, Villalobos-Garita A, Ramírez-Luna MÁ. Use of añovel covered self-expandable metal stent with an anti-migration system for endoscopic ultrasound-guided drainage of a pseudocyst. World JGastrointest Endosc. 2013;5(6):297-9.

21. Deviere J, Devaere $S$, Baize $M$, et al. Endoscopic biliary drainage in chronic pancreatitis. Gastrointest Endosc. 1990;36:96-100.

22. Catalano MF, Linder JD, George S, et al. Treatment of symptomatic distal common bile duct stenosis secondary to chronic pancreatitis: comparison of single vs multiple simultaneous stents. Gastrointest Endosc. 2004;60(6):945-52.

23. Arslanlar S, Jain R. Benign biliary strictures related to chronic pancreatitis: Balloons, stents, or surgery. Curr Treat Options Gastroenterol. 2007;10:369-75. 